\title{
Observations and modelling of non-proportional mixed mode cyclic loading
}

\author{
Michael Vormwald ${ }^{1, *}$ \\ ${ }^{1}$ Materials Mechanics Group, Technische Universität Darmstadt, Germany
}

\begin{abstract}
Under non-proportional mixed-mode loading conditions at the crack tip, some observations are reported. They are limited to cases of cylindrical specimens under normal force and torsion. The fatigue cracks have been initiated by the same loading for which the crack growth behavior has been studied. The observations are discussed against the background of a variety of influence factors on non-proportional mixed mode growth. Finally, a couple of modeling approaches are listed which are - according to the nature or the problem - not yet developed for providing prediction tools in an engineering environment.
\end{abstract}

\section{Introduction}

The calculation of fatigue crack growth along a predefined path is state of the art, see for example one of the latest guidelines [1]. The cyclic crack propagation can be calculated with proportional loading, based on the criterion of local symmetry $\left(K_{\mathrm{II}}=0, K_{\mathrm{III}}=0\right)$, even with developing continuously curved crack surfaces. The analysis of such problems requires the simulation of load-bearing and structural geometry-compatible Mode I crack geometry. For plane problems, suitable software is developed and widely applicable [2]. In the simulation of three-dimensional Mode I crack growth, application-oriented concepts [3, 4] are often used, which generally do not reflect the phenomenon of multiple initial single-cracking [5] at the crack front.

Non-proportionality itself, in general, is a matter of loading sequences acting on structures. Several, at least two load sequences that are acting independently are required to cause significant local non-proportional stress states or - in case of cracked structures - nonproportional mixed-mode loading conditions. Fatigue crack growth assessment under these conditions is in the fledgling stages. The fracture mechanics guidelines do not address nonproportional loading conditions. In a narrower sense, they are understood as cases when during a time period of loading (a cycle, howsoever it is identified) the ratios of mode-related crack driving force parameters (e.g. $\Phi=K_{\mathrm{II}} / K_{\mathrm{I}}$ ) vary significantly.

Figure 1 shows a collection of variables influencing the direction and the increment of crack growth. They will be discussed in the next section in some more detail. In Sect. 3 observations of laboratory-analyzed findings gathered during the past decades are presented. Modeling efforts are indicated in Sect. 4 before the contents are summarized. For a comprehensive access to the subjects of non-proportionality and mixed mode loading please see some recently published overview articles [6-8].

\footnotetext{
*e-mail: vormwald@wm.tu-darmstadt.de
} 


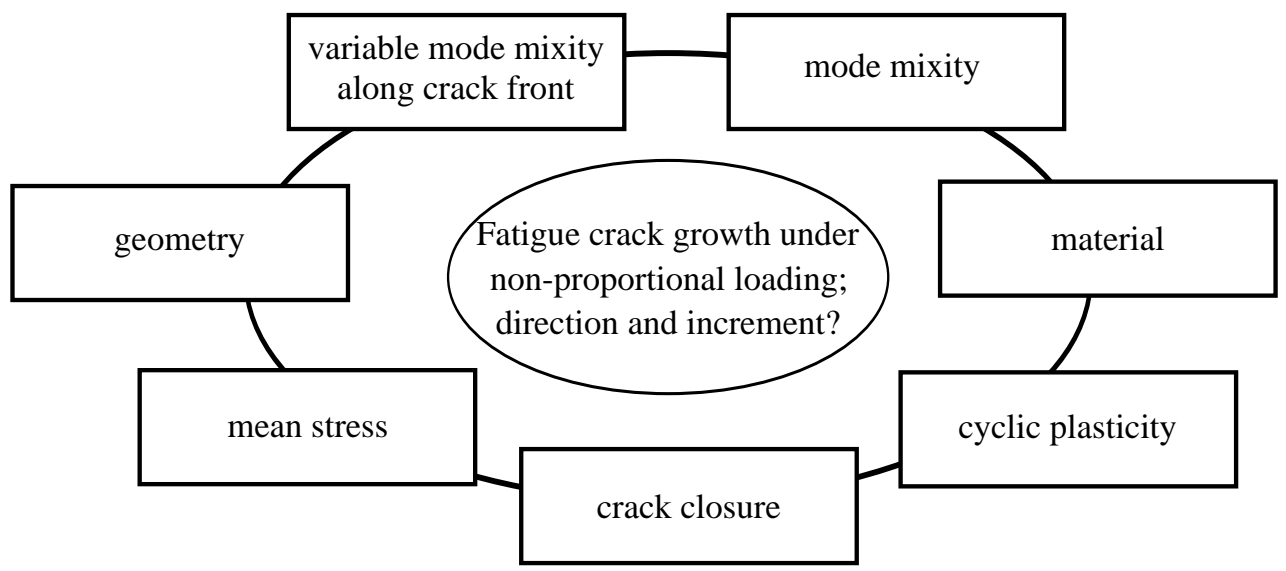

Figure 1. Major variables for fatigue crack growth under non-proportional mixed mode conditions

\section{Major variables for fatigue crack growth under non-proportional mixed mode}

For the description of the crack evolution during the fatigue crack growth stage two points are of interest: the crack path and the crack growth rate. For each increment, length and direction must be evaluated. Both items have shown to depend on various influence factors while the crack tip or the crack front are subjected to non-proportional mixed mode loading conditions.

As $\Phi$ varies during a load cycle, it is inevitable that any direction of crack advance will again lead to a new situation showing non-proportional mixed mode. The new situation may be assessed whether a time-averaged Mode II course gives higher or lower values. If the crack path develops such that the Mode II (and eventually Mode III) course is minimized, the crack's behavior can be called tensile crack growth. Oppositely, if the Mode I course is minimized the behavior can be called shear crack growth. It is worth noting that pure tensile or shear crack growth situations do not exist, except in some special cases mentioned in a later section. A self-intensifying behavior exists: a tensile crack prefers to remain a tensile crack, a shear crack prefers to grow under dominating shear. A certain kind of inertia seems to exist.

However, this inertial behavior is disturbed by increasing cyclic plasticity. As cyclic plastic deformation tends to develop in directions where shear stresses are large, the cracks like to follow the associated planes. In the early stages of crack initiation, the cracks form along planes of maximum shear amplitudes, in the final stages of a pure Mode I, tensile crack in a plate, the surface develops slanting to a mixed Mode I and III situation driven by cyclic shear bands crossing the plate. These two examples should make clear that shear cracks are a common observation also in proportional loading situations. Shear crack growth is more frequent in the non-proportional situation. The way how the material develops its slip system seems to play a role, however, no clear classification is available today with respect to which materials show preferred obedience to tensile or shear crack growth behavior. An eventually existing anisotropy will influence the behavior, too.

Cyclic plasticity causes plasticity induced crack closure. In the non-proportional mixed mode situation the roughness induced crack closure appears on top and becomes more relevant the more shear crack growth appears. The phenomenon interacts with the plasticity induced crack closure. Roughness is 'produced' in loading with small amplitudes and preferred 
tensile loading. Mode II kinks, even if of short length, cause roughness induced closure, leading to an additional crack tip shielding and decelerated growth. Under high amplitudes and considerable shear the crack surface is abraded, roughness and plasticity induced closure are decreased, leading to an accelerated growth. Even on top of this already complex context, curved and warped crack surfaces give reason to a long-range interlocking.

Crack closure itself, especially plasticity induced crack closure, strongly depends on mean loads or $R$-ratios. Any kind of closure will disappear at high $R$-ratios, whereas for alternating loads for example the modeling of closure in non-proportional loading causes severe trouble. For pure Mode I tensile crack growth, the mean load (or $R$-ratio) dependency of crack growth rates can nearly completely be explained by plasticity induced crack closure, see e.g. [9]. In non-proportional mixed mode loading explanations might follow similar arguments, however, the topics of roughness and long-range curvature (geometry) induced closure hinder a straight forward approach.

The geometry of the structure, even the uncracked geometry, plays a role in the growing crack's search for its path. For example the crack might want to grow in a tensile mode. The small cross section, the ligament, might, however, be oriented such that a shear dominated crack is enforced. Obviously, this geometry influence is related to cyclic plasticity because in the small cross section the largest plastic deformation is to be expected to occur.

Finally, in three-dimensional structures the mode-mixity is generally changing along the crack front. The direction of the crack growth increment along the crack front according to a criterion identified under plane stress or strain conditions might lead to incompatible crack surface geometries. Stepped and fissured cracks evolve.

These influence factors shown in figure 1 are interrelated. Focusing on individual aspects, although preferable from a scientific point of view, is hard to achieve.

\section{Observations}

The current paper can give not even an only limited overview of non-proportional mixed mode fatigue crack growth. A mostly subjective choice of results is gathered instead with a focus on own research during the past decades.

Experimental investigations of the fatigue strength of non-proportionally loaded structures are common state of the art. The action of various load sequences in their realistic interconnection is reproduced in a laboratory. In numerical fatigue life assessments, methods for dealing with the initiation of fatigue cracks are available even for the complicated non-proportional cases of combined cyclic loading [6]. The theoretical and practical support is immediately decreasing as soon as the growth of initiated fatigue cracks under nonproportional cyclic loading conditions is a matter of concern. The topic of non-proportional mixed mode fatigue crack growth has, however, become a field of scientific interest. Within this field it is of paramount interest to study the behavior of cracks having initiated 'naturally' under fatigue loading at structural notches. The initiation itself can even occur under local uniaxial (and therefore locally proportional) conditions. This situations regularly appears in thin-walled structures.

Zerres et al. $[10,11]$ investigated such cracks in thin-walled tubes initiated at a starter notch under non-proportional normal force and torsion. The experiments have been carried out by Brüning [12]. The specimen is shown in figure 2. The specimens were loaded under alternating $(R=-1)$ combined $90^{\circ}$-out-of-phase cyclic normal force and torsion. Different crack paths developed at different loading amplitudes, shown in figure 3. The number of cracks depends on the force $F$ to moment $M$ ratio. High torsion drives four cracks, dominating force drives two cracks. Considerable crack surface warping is only observed for the case with the lowest amplitudes. 
a) Specimen

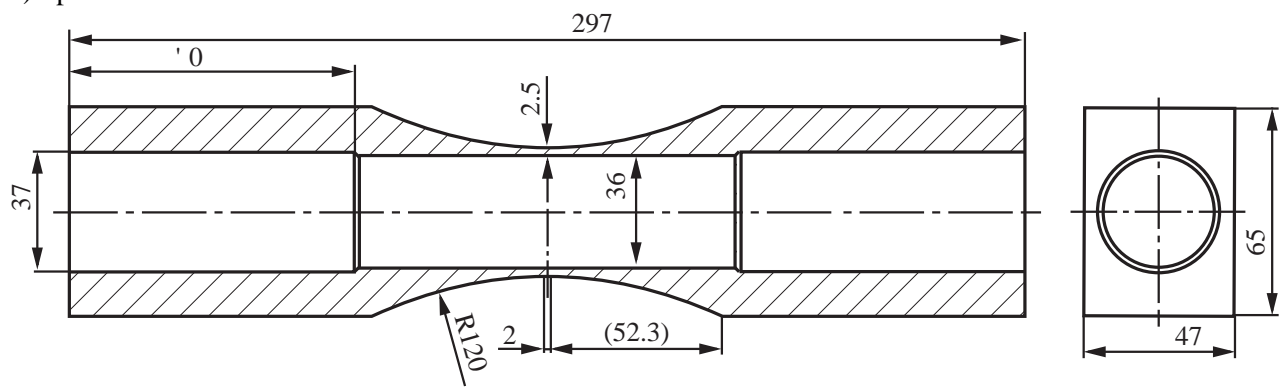

b) Crack length definition

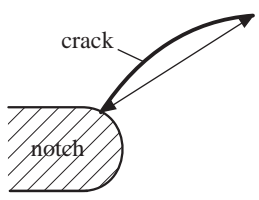

c) Sketch of fracture surface indicating corner crack

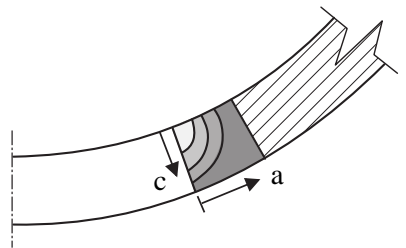

Figure 2. Specimen shape, dimensions in millimeters

Besides testing thin-walled tubes, Highsmith [13] also performed experiments with inclined through-crack round (ITCR) specimens. While in thin-walled tubular specimens the mode-mixity is nearly constant along the crack front (its length is nearly equal to the tube's wall thickness), it varies considerably along the crack front in ITCR-specimens. Regardless of which criterion the crack growth might want to obey, the direction of crack advance differs between neighboring crack front locations. This gives rise to either warped crack surfaces or fraying of the crack front, or both.

Due to the hourglass-shaped specimen geometry shown in figure 2 any crack path trying to escape from the center was suspect of being bent back to the net cross section. Therefore, another test series with constant cross section tubular specimens was performed by Hos [14]. The outer and inner diameters or the circular ring cross section were $d_{o}=42.4 \mathrm{~mm}$ and $d_{i}=37.2 \mathrm{~mm}$. In more than 50 experiments the applied load sequence was varied including variations of the phase shift, the $R$-ratio, the ratio of force to moment, and the load amplitudes. In addition, tests with load sequences with different frequencies of the load channels, variable amplitude etc. were performed. The crack tip coordinates and associated cycle numbers are recorded in reference [14]. Figure 4 shows an example for crack paths under phase shift of $90^{\circ}$. The paths are similar to those obtained by Brüning, indicating that Brüning's nonuniform cross section had only minor influence on the path geometry.

A few of these tests have been interrupted periodically. For a couple of cycles, the deformation field near the crack tip has been measured applying the digital image correlation technique. Figure 5 shows an example for the case with a phase angle of $90^{\circ}$ and $R=-1$. Further information can be found in reference [15]. At the top the normal strain component $\varepsilon_{y y}$ is shown, at the bottom the shear-related tensor component $\varepsilon_{x y}$ appears. The two snapshots belong to the same cycle, however, they are taken at different instants of time during the cycle. The origin of the coordinate system is located at the crack tip. The measurements have been performed shortly before the crack path kinked to the right. Both components $\varepsilon_{y y}$ and $\varepsilon_{x y}$ reveal their angular maximum pointing in the direction of the path development to come. 


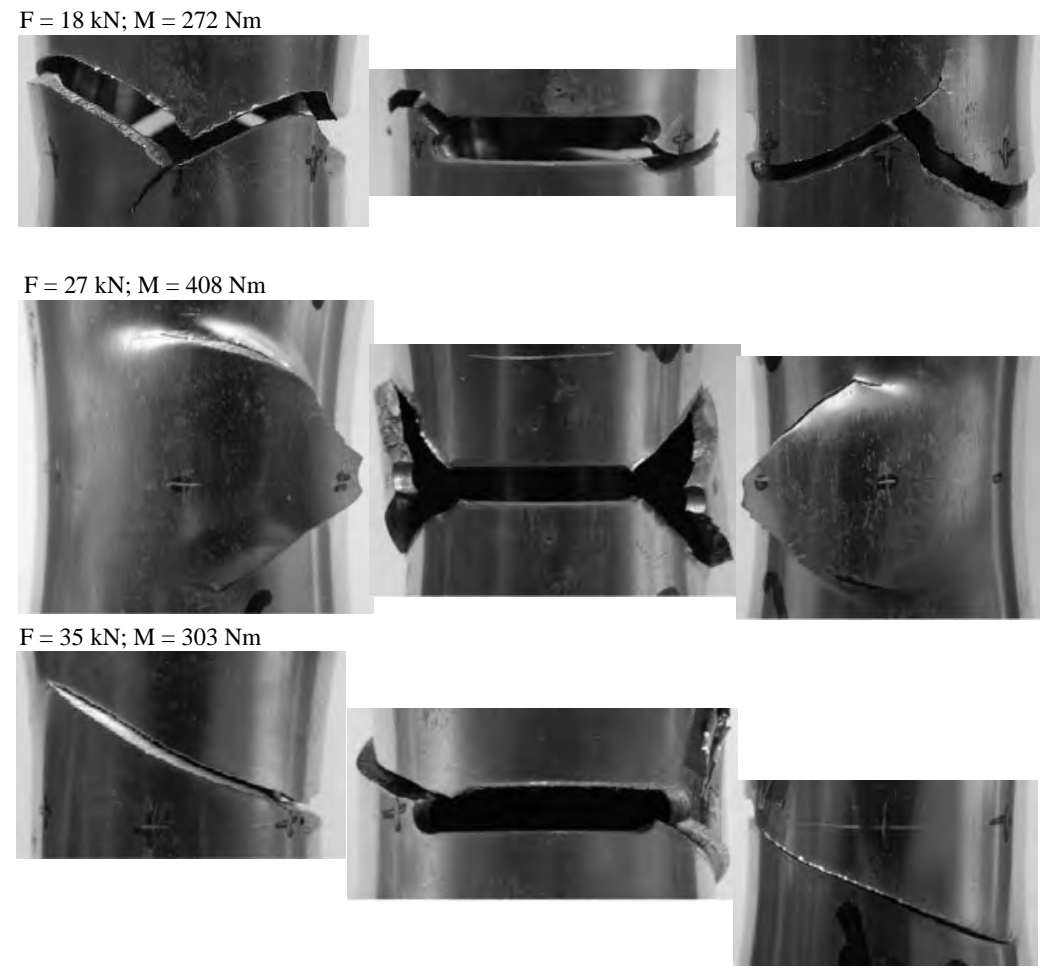

Figure 3. Crack path under out-of-phase loading with a phase angle of $90^{\circ}$, after [12]
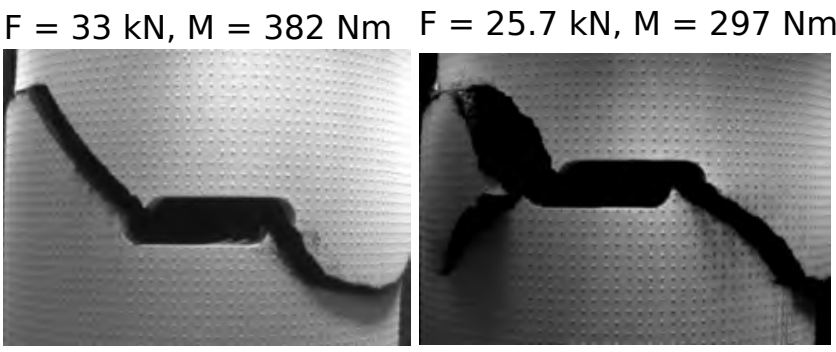

Figure 4. Crack path under out-of-phase loading with a phase angle of $90^{\circ}$, according to [14]

A tensile as well as a shear crack growth demands for a kink - and it occurred. This means, however, that a clear distinction concerning the type of growth cannot be made. Another observation can be made by digital image correlation which is obvios in the macroscopic scale also in figures 3 and 4: there is a Mode III component cyclically operating at the crack front and leading to a Mode III-ratcheting which continiously bends shell shingles outward. The collection of observation presented so far might have made clear that the topic under examination hides many secrets to be discovered. At the time being, direct observation of the deformation processes seem to be the most promising way for increasing insight into a unsolved, however, fundamental issue of fatigue. Observation alone is not constructive enough. It ought to be accompanied by modeling effort. 


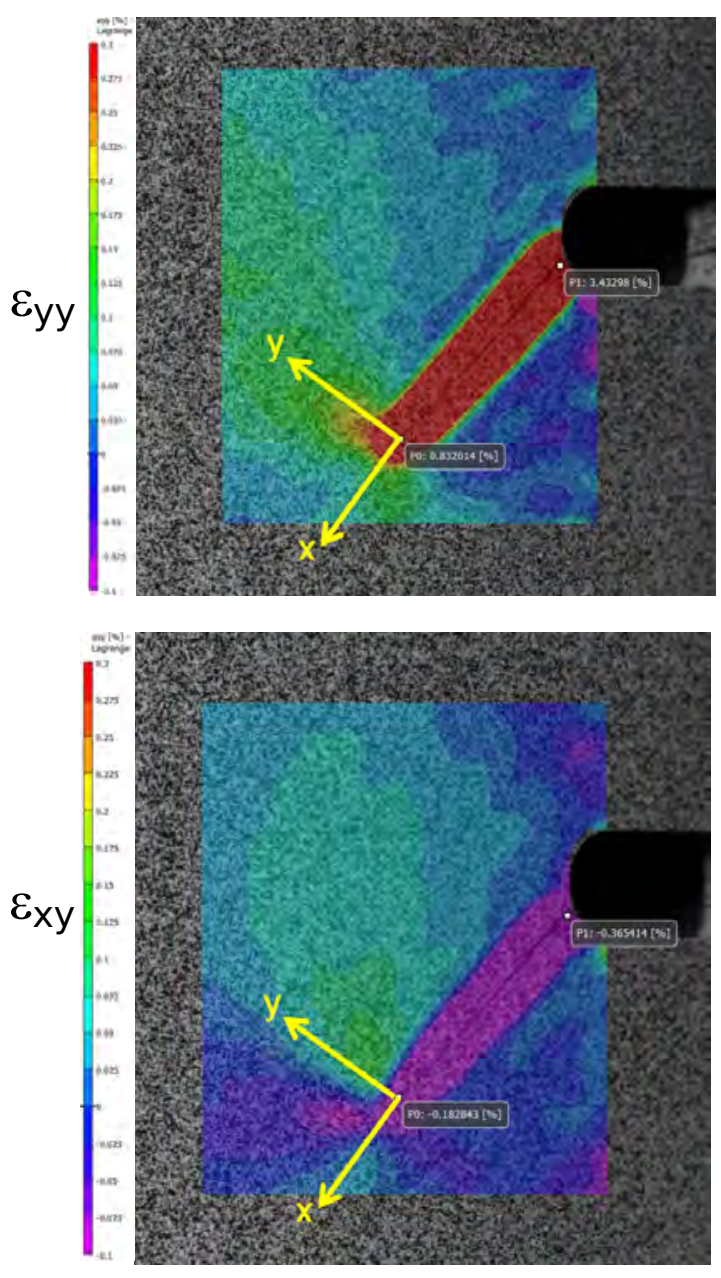

Figure 5. Strain field snap shots of the crack region under out-of-phase loading with a phase angle of $90^{\circ}$, according to [14]

\section{Modeling}

In her crack growth modeling, Brüning [12] assumed that the cracks grow in a plane, the orientation of which is determined by crack initiation. Crack initiation occurred at a location with maximum local stress and strain amplitudes, see figure 3. At the instant of time when the local stress reaches its maximum value, both loading channels contribute to these stresses. Fatigue crack growth has been calculated with the constant, frozen values of force and moment values interpreted as amplitudes. A plasticity correction was applied via determining the cyclic effective $J$-integral. Crack closure was taken into account by estimating opening loads with Newman's formula [16]. Calculated and measured crack growth curves are compared in figure 6. Numerical results have been only partly acceptable. As expected, the cases with large path curvature could not be modeled well. In addition, the crack growth rate was underestimated for high amplitudes.

Yang and Vormwald $[17,18]$ re-analyzed some of Brüning's results. The crack path curvature was allowed. However, the algorithm was based on linear elastic fracture mechanics. 

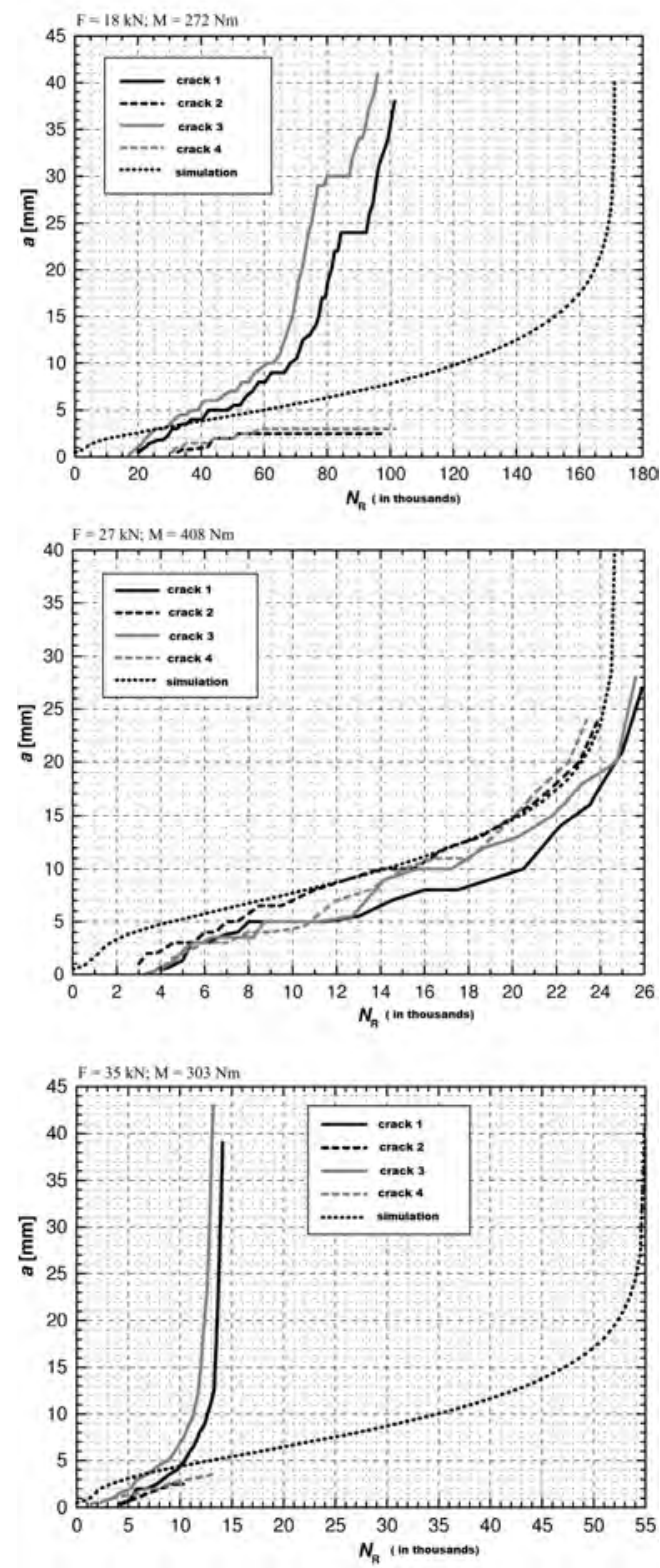

Figure 6. Comparison of crack growth curves for the cases shown in figure 3, according to [12]

Therefore, only cases with low amplitudes have been considered. Various mixed mode fracture criteria have been applied to estimate the crack growth direction with a focus on the maximum tangential stress (MTS) criterion. The maximum shear stress criterion (MSS) is used as an alternative. The simulation results including the crack growth paths as well as the fatigue life assessment are compared with the results from the experimental investigation. The experimentally determined path has been modeled, too. An example is shown in figure 7. During the first stage with a nearly straight path the growth behavior can be modeled nicely 

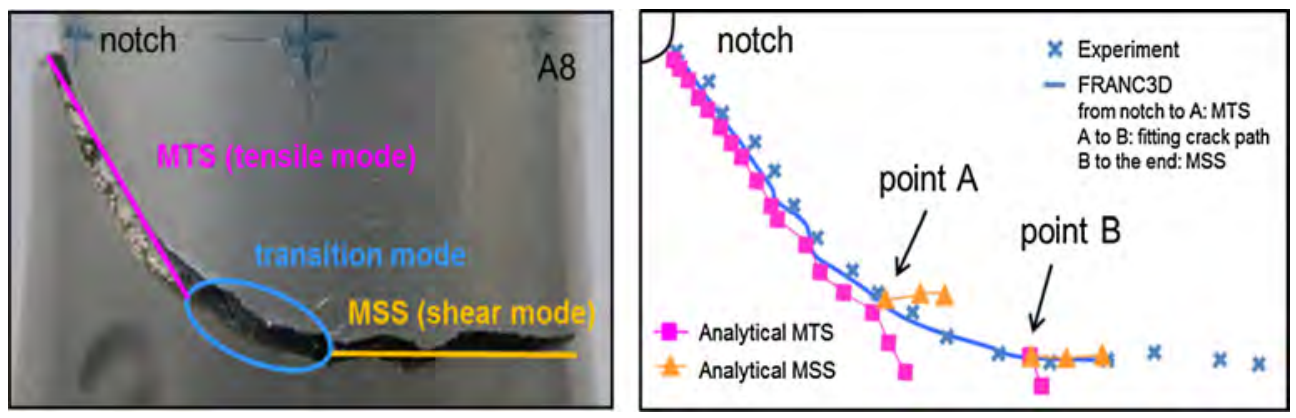

Figure 7. Comparison of crack growth paths, experiment according to [12], calculations according to [17]

by the maximum tangential stress hypothesis. The path deviates from the MTS-predicted path at a later stage. At the path's knee point, the predicted orientation according to the MSS criterion is also shown in figure 7. A fracture mode transition from tension to shear is observed. In some specimens, see [17], three distinct fracture modes are observed: tensile mode, shear mode and transition mode. The crack path in transition mode cannot be predicted correctly, neither by the MTS criterion nor by the MSS criterion. Preliminarily, applying both criteria is proposed, accompanied by an engineering judgement of the consequences of choosing either of the criteria. As also shown in figure 7, at the final stages of crack growth, both predicted paths do not necessarily deviate much. If both criteria indicate the same direction, the maxima of tangential and shear stress occur at the same angle of the singular crack tip stress field, however, at different times during the non-proportional cycle. The crack growth curves belonging to the path shown in figure 7 are presented in figure 8 . The simulation accuracy is better than acceptable in this individual case.

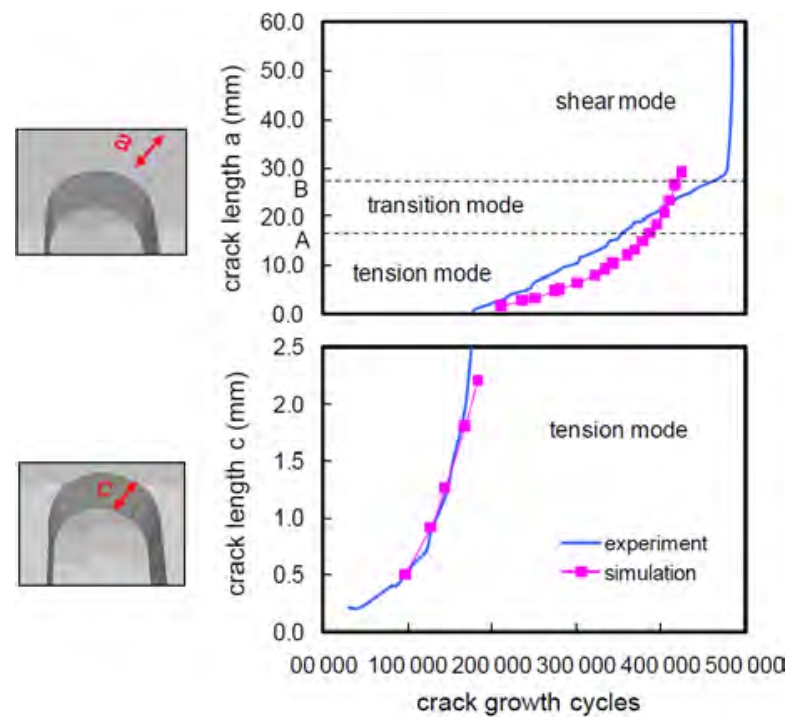

Figure 8. Example of a crack growth curve, experiment according to [12], calculations according to [17] 
While examining the history of stress components, Yang took advantage of the fact that peak values and amplitudes of the component under consideration turn towards the same direction due to cyclic loading with $R=-1$. Generally, a decision which of both values, or which combination, is declared decisive for the crack growth direction. In this context, Highsmith [13] observed that crack angles generally fall between the maximum of some equivalent stress intensity factor $K_{\text {eq, max }}$ and its range $\Delta K_{\text {eq }}$. The applied mixed mode hypothesis determines what should be understood as equivalent. He suggest that the crack driving force $\Delta \bar{K}$ accounts for both, the maximum and the range,

$$
\Delta \bar{K}=\Delta K_{\mathrm{eq}}^{w} K_{\mathrm{eq}, \max }^{(1-w)} .
$$

A first estimate of $w=0.3$ was published for tensile crack growth, $w=0.7$ should be preferred for shear crack growth in the nickel based super alloy of Highsmith's investigation. This phenomenological description of the mean load influence has not been related to crack closure. Oppositely, Brüning [12] and Yang [17] used Newman's crack opening estimate for identifying effective ranges, thus taking the mean load effect into account.

In continuation of previous work, Hos [14, 19] calculated opening and closing events applying the finite element (FE) technique. FE-models have been created resembling the crack geometry observed in the experiment. Contact elements were arranged at the crack surface, their status indicated events of opening and closing. A node release scheme was applied which under Mode I conditions would nicely predict plasticity induced closure in combination with an adequate plasticity model. The friction coefficient of the contact elements was set to zero. Roughness induced closure was therefore neglected. Having identified the portions of the cycle when the crack is open, the cyclic $\Delta J$-integral was determined numerically with a specifically developed post-processing software. For the details of the $\Delta J$-calculation procedure see [20]. Figure 9 shows a comparison of crack growth rates. It turned out that for the crack growth rates obtained in the experiment, the numerically calculated $\Delta J_{\text {eff }}$ were too small by a factor of 2 ; in other words, a calculated $\Delta J_{\text {eff- }}$-value would underestimate the crack growth rate by a factor of 3 . This shows that the development of a prediction tool has not yet come to a satisfying solution. In addition, further developing a $\Delta J_{\text {eff }}$-based tool will require inventing a hypothesis for predicting the growth direction. The latter certainly requires a splitting up of $\Delta J_{\text {eff }}$ into its mode-related components. However, the last sentences belong to the outlook.

Whether or not a mixed mode criterion describes crack growth behavior realistically can also be assessed by observing the near crack tip strain field - on top of recording the crack path. Gonzáles et al. [21] presented such evaluations based on measurements using digital image correlation. They used the Westergaard solution to describe the strain state near the crack tip for correlating the measured displacements in the crack wake near the crack tip with the stress intensity factors under Mode I and Mode II. In the measured displacements, both elastic and plastic portions of the deformation are included. The derived stress intensity factors therefore comprise a plasticity correction which should be reasonable as long as the elastic singular stress field is dominating. Maybe it is wasting words mentioning that also crack closure is contained and the resulting stress intensity factors are effective ranges $\Delta K=$ $\Delta K_{\text {eff }}$ when taking the difference of values belonging to reversal points. The evaluations have been extended in [15] for incorporating also the Mode III components. The stress intensity factors have been inserted in the mixed mode criterion of Schöllmann et al. [22],

$$
\Delta K_{\mathrm{eqS}}=\frac{1}{2} \cos \frac{\theta}{2}\left[\Delta K_{\mathrm{I}} \cos ^{2} \frac{\theta}{2}-\frac{3}{2} \Delta K_{\mathrm{II}} \sin \theta+\sqrt{\left(\Delta K_{\mathrm{I}} \cos ^{2} \frac{\theta}{2}-\frac{3}{2} \Delta K_{\mathrm{II}} \sin \theta\right)^{2}+4 \Delta K_{\mathrm{III}}^{2}}\right] .
$$




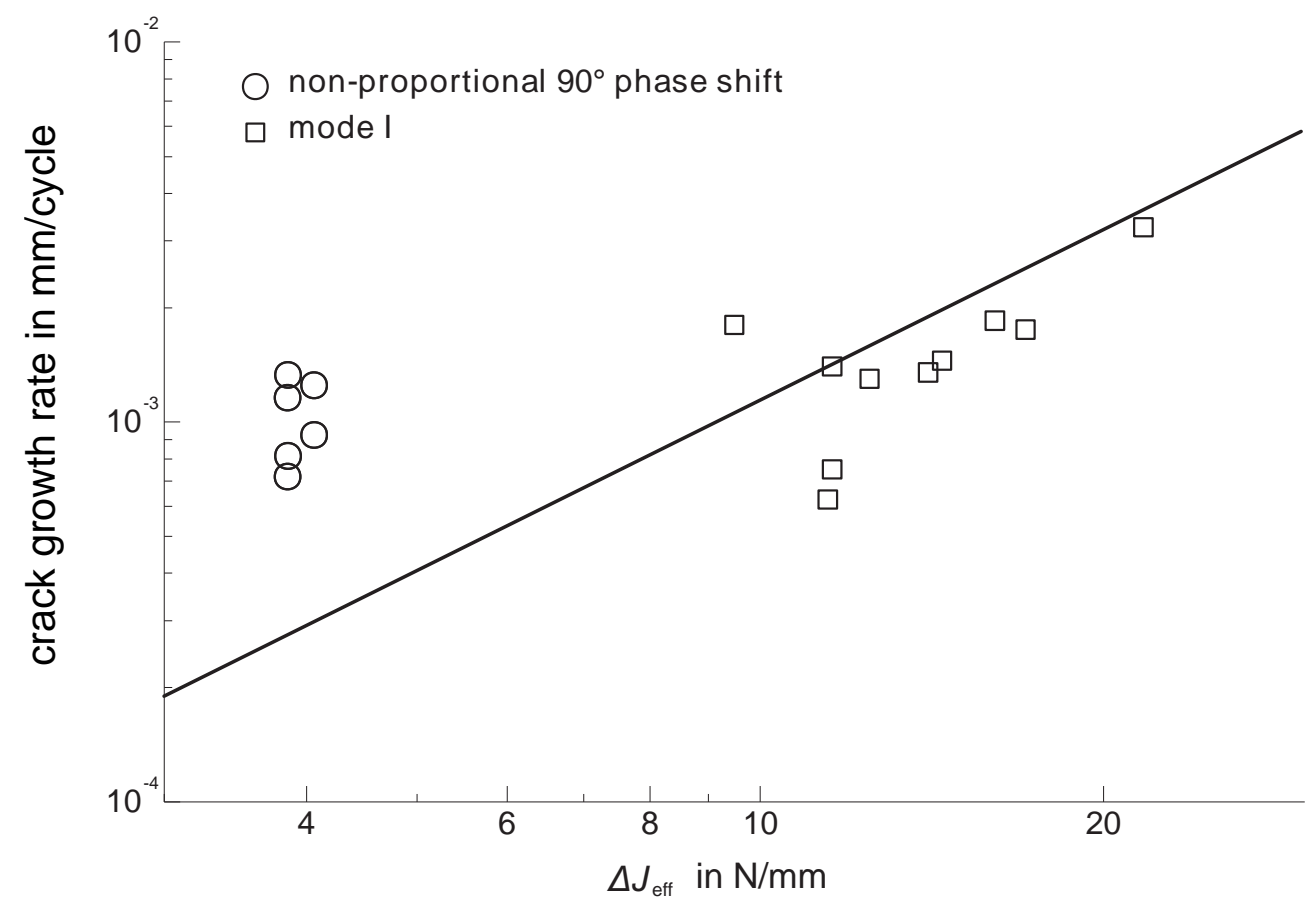

Figure 9. Experimentally determined scatter bands of crack growth rates under non-proportional mixed mode plotted against the numerically calculated $\Delta J_{\text {eff }}$ according to [14]

When mode-mixities change inside one cycle, it is required to find a maximum range value of $\Delta K_{\text {eqS }} \rightarrow \Delta K_{\text {eqS,max }}$ that would occur during one cycle by searching which angle $\theta_{\text {max }}$ would lead to the maximum. The latter angle points to the direction of the crack growth increment.

In figure 10 the crack growth rate is plotted over the equivalent range $\Delta K_{\text {eqS, max }}$. In an ideal situation, the data points obtained would fall into a narrow scatter band. The width of the present scatter band is a factor of 8 in crack growth rate direction. Although some scatter is due to the experimental technique, a portion has its origin in the mixed mode hypothesis because it might not describe the fatigue crack growth behavior perfectly. Against this background, the factor of 3 in growth rates - as seen in figures 6 and 9 - when predicted by applying $\Delta J_{\text {eff }}$-based estimates of growth rates does no longer appear to be too far out of range.

For cases for which a hypothesis is shown to be valid, the modeling of fatigue crack growth requires to take all phenomena collected in section 2 and shown in figure 1 into account. Above all, crack closure and cyclic plasticity is of paramount importance. In a finite element environment the mesh must be updated including the crack growth increment which is to be determined by the mixed mode hypothesis. A new FE-model must be created for every crack increment. The deformation and the internal variables of the plasticity model left in the previous FE-model must be transformed to the new FE model. The numerical effort is enormously high. This can be concluded from first investigations of such a kind of modeling performed by Zerres et al. [23]. 


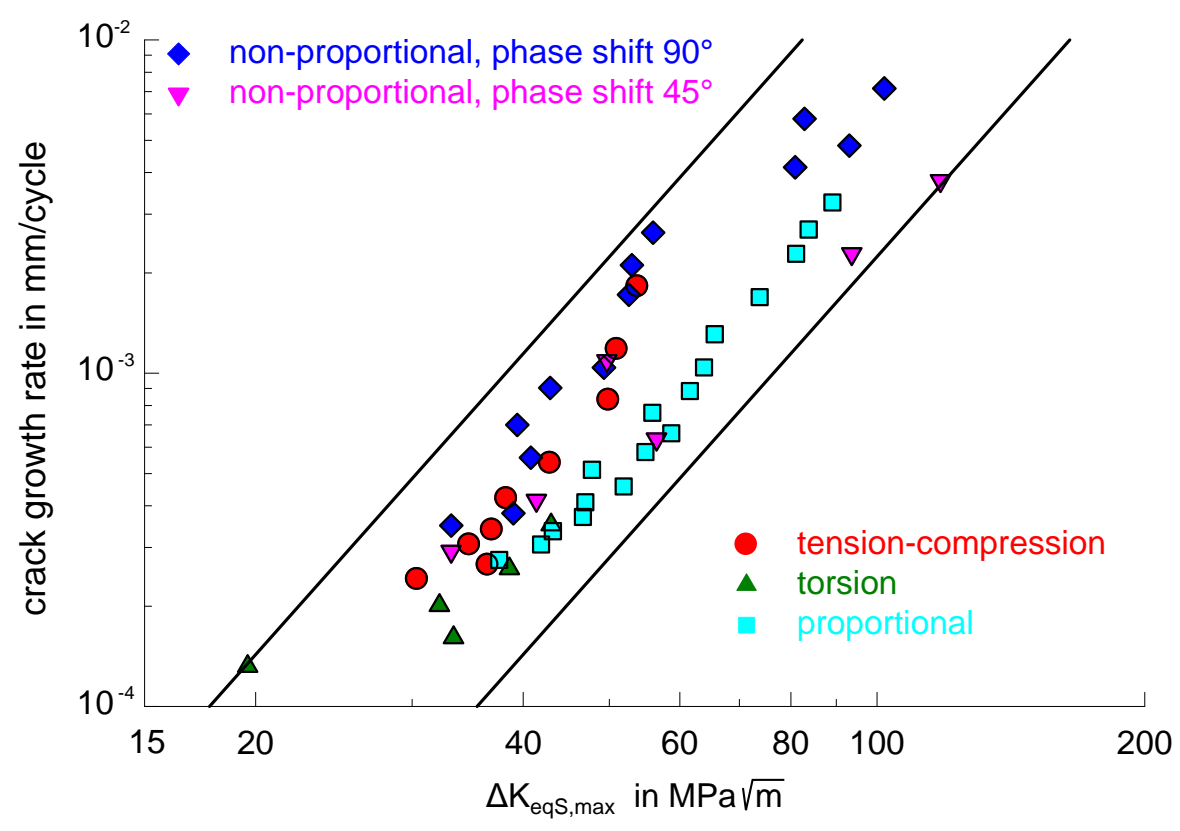

Figure 10. Correlation of fatigue crack growth rates with Schöllmann's equivalent stress intensity factor range, extended for application with non-proportional mixed mode according to [15]

\section{Summary}

Based on observations reported in this paper, it can be concluded that fatigue crack growth under non-proportional mixed mode loading is depending on various influence factors, which are interrelated and can hardly be separated for individual investigations. Mode-mixity, which can also vary along three-dimensional crack fronts, plays an important role concerning the crack growth behavior in a way that for high mode-mixities a tendency to shear dominated crack growth can be observed, whereas for low mode-mixities Mode I dominated crack growth is observable. However, this change in the crack growth behavior was also found to be strongly material, (cyclic) plasticity and geometry dependent. By looking at the fatigue crack growth rates, crack closure phenomena play a key role. Under non-proportional mixed mode loading an interaction between plasticity induced crack closure (resulting from the cyclic plastic deformation), roughness and friction induced crack closure is identifiable. This interaction can lead to both, accelerated and decelerated growth rates. This strength of interaction is in turn strongly dependent on the loading level, especially the mean stress.

In order to predict the crack growth behavior under non-proportional loading, several criteria have been proposed. However, due to different shortcomings of all the criteria, today, no criterion for general usage under non-proportional loading is available. Nevertheless, as best craftsmanship, a prediction trial should be based on the maximum tangential stress hypothesis where it has to be decided whether amplitudes or maxima or a combination thereof is declared responsible for direction and increment. An application of a variant of the maximum shear stress hypothesis might be an amendment. In the probable case of significant crack curvature, crack face contact should be considered. This means that contact elements have to be inserted between opposite crack faces. This operation together with the necessary remeshing after a numerical crack growth increment requires large computing capacities. An even stronger demand stems from the necessity to consider non-linear deformation behavior. 
The stress intensity factors have to be replaced by crack driving forces defined in terms of the elastic-plastic fracture mechanics. The near crack front opening displacements are a candidate as well as some further developments of the J-integral. However, considering non-linear deformation behavior - no matter with which mixed mode hypothesis - requires that the previous history of the plastic deformation must be restored after remeshing. Summarizing, fatigue crack growth assessment under non-proportional loading conditions is in the fledgling stages.

\section{References}

[1] C. Berger, J.G. Blauel, L. Hodulak, B. Pyttel, I. Varfolomeev, Bruchmechanischer Festigkeitsnachweis (VDMA-Verlag Frankfurt, $4^{\text {th }}$ edition, 2018) 368 pages

[2] H. Theilig, M. Wünsche, R. Bergmann, Numerical and experimental investigation of curved fatigue crack growth under proportional cyclic loading Steel Research International 74, 566-576 (2005)

[3] G. Dhondt, Cutting of 3D FE mesh for automatic mode I crack propagation calculation International Journal of Numerical Methods in Engineering 42, 749-772 (1998)

[4] M. Schöllmann, M. Fulland, H.A. Richard, 3D fatigue crack growth simulation under complex loading with ADAPCRACK3D ESIS Publication 26, paper 5, 1-8, (2000)

[5] M. Ressel, H. Theilig, Finite Element Analysis of Secondary Cracks at a Crack Front under Pure Mode III Loading Key Engineering Materials 488-489, 605-608 (2012)

[6] A. Fatemi, N. Shamsaei, Multiaxial fatigue: An overview and some approximation models for life estimation International Journal of Fatigue 33, 948-958

[7] P. Zerres, M. Vormwald, Review of fatigue crack growth under non-proportional mixedmode loading International Journal of Fatigue 58, 75-83 (2014)

[8] M. Vormwald, O. Hertel, P. Zerres, Fatigue of engineering structures under combined nonproportional loads: An overview Fatigue \& Fracture of Engineering Materials \& Structures 41, 1449-1468 (2018)

[9] M. Vormwald, Ermüdungslebensdauer von Baustahl unter komplexen Beanspruchungsabläufen am Beispiel des Stahles S460 Materials Testing 53, 98-108 (2011)

[10] P. Zerres, J. Brüning, M. Vormwald, Fatigue crack growth behavior of fine-grained steel S460N under proportional and non-proportional loading Engineering Fracture Mechanics 77, 1822-1834 (2010)

[11] P. Zerres, J. Brüning, M. Vormwald, Crack Growth Behaviour of the Aluminium Alloy AlMg4.5Mn at Proportional and Non-Proportional Fatigue Loading Materials Testing 53, 109-117 (2011)

[12] J. Brüning, Untersuchungen zum Rissfortschrittsverhalten unter nichtproportionaler Belastung bei elastisch-plastischem Materialverhalten - Experimente und Theorie (Inst. Stahlbau u. Werkstoffmechanik TU Darmstadt, report 85, 2008) 339 pages

[13] J. Highsmith (2009), Crack path determination for non-proportional mixed-mode fatigue (Ph.D. thesis, Georgia Institute of Technology, USA) (2009) 249 pages

[14] Y. Hos, Numerical and experimental investigation of crack growth in thin-walled metallic structures under nonproportional combined loading (Inst. Stahlbau u. Werkstoffmechanik TU Darmstadt, report 114, 2017) 249 pages

[15] M. Vormwald, Y. Hos, J.L.F. Freire, G.L.G. Gonzáles, J.G. Díaz, Crack tip displacement fields measured by digital image correlation for evaluating variable mode-mixity during fatigue crack growth International Journal of Fatigue 115, 53-66 (2018)

[16] J.C. Newman Jr., Crack opening stress equation for fatigue crack growth Journal of Fracture 24, R131-R135 (1984) 
[17] Y. Yang, LEFM-based simulation of fatigue crack growth under non-proportional mixed-mode loading (Inst. Stahlbau u. Werkstoffmechanik TU Darmstadt, report 105, 2013) 128 pages

[18] Y. Yang, M. Vormwald, Fatigue crack growth simulation under cyclic non-proprtional mixed mode loading International Journal of Fatigue 102, 37-47 (2017)

[19] Y. Hos, M. Vormwald, Experimental study of crack growth under non-proportional loading along with first modeling attempts International Journal of Fatigue 92 426-433 (2016)

[20] O. Hertel, R. Döring, M. Vormwald, Cyclic J-integral under nonproportional loading Proceedings of the 7th international conference on biaxial/multiaxial fatigue \& fracture, DVM/ ESIS, 513-518 (2004)

[21] G.L.G. Gonzáles, J.G. Diaz, J.A.O. González, J.T.P. Castro, J.L.F. Freire, Determining SIFs Using DIC Considering Crack Closure and Blunting Experimental and Applied Mechanics 4, 25-36 (2017)

[22] M. Schöllmann, H.A. Richard, G. Kullmer, M. Fulland, A new criterion for the prediction of crack development in multiaxially loade structures International Journal of Fracture 117, 129-141 (2002)

[23] P. Zerres, M. Vormwald, Finite element based simulation of fatigue crack growth with a focus on elastic-plastic material behavior Computational Materials Science 57, 73-79 (2012) 